\title{
The effect of diabetes mellitus on
}

Ozlem Equils $^{1 *}$, Christopher da Costa ${ }^{1}$, Michele Wible ${ }^{1}$ and Benjamin A. Lipsky ${ }^{2,3}$

\begin{abstract}
Background: The presence of diabetes mellitus increases the risk of several severe infections, but data on its effect on treatment outcomes in patients with nosocomial pneumonia (NP) caused by methicillin-resistant Staphylococcus aureus (MRSA) are limited.

Methods: We retrospectively analyzed data from a double-blind, randomized, multi-center, international clinical trial of culture-confirmed MRSA NP that compared treatment with linezolid to vancomycin. Specifically, we evaluated the clinical and microbiologic outcomes of patients with and without diabetes in the modified intent to treat population at end-of-treatment (EOT) and end-of-study (EOS, 7-30 days post-EOT).

Results: Among 448 enrolled patients 183 (40.8\%) had diabetes mellitus, 87 (47.5\%) of whom received linezolid and 96 (52.5\%) vancomycin. Baseline demographic and clinical characteristics were similar for the two treatment groups. Clinical success rates at EOS were $57.6 \%$ with linezolid and $39.3 \%$ with vancomycin, while microbiological success rates were $58.9 \%$ with linezolid and $41.1 \%$ with vancomycin. Among diabetic patients, rates of mortality and study drug-related adverse effects were similar between the treatment groups. Overall day 28 mortality rates were higher among diabetic patients compared to non-diabetic patients ( 23.5 vs $14.7 \%$, respectively: RD $=8.8 \%$, $95 \% \mathrm{Cl}[1.4,16.3])$.
\end{abstract}

Conclusions: Among diabetic patients with MRSA NP, treatment with linezolid, compared to vancomycin, was associated with higher clinical and microbiologic success rates, and comparable adverse event rates.

Trial registration: NCT00084266.

Keywords: Linezolid, Diabetes mellitus, MRSA, Vancomycin, Pneumonia, Mortality, Outcome, Staphylococcus, Infection, Prognosis

Abbreviations: AE, Adverse events; ELF, Epithelial lining fluid; EOS, End-of-study; EOT, End-of-treatment; (Continued on next page)

\footnotetext{
* Correspondence: Ozlem.equils@pfizer.com

${ }^{1}$ Pfizer Inc., Collegeville, PA, USA

Full list of author information is available at the end of the article
} 
(Continued from previous page)

ICUs, Intensive care units; IDSA, Infectious Diseases Society of America; MIC, Near zero variance, minimum inhibitory concentration; MITT, Modified-intent-to-treat population; MRSA, Methicillin resistant Staphylococcus aureus;

NP, Nosocomial pneumonia; SAEs, Serious adverse events

\section{Background}

Persons with diabetes mellitus, compared with nondiabetic persons, have higher rates of impaired immunity $[1,2]$, decreased lung function $[3,4]$, and an increased risk for various types of infection, including pneumonia $[5,6]$. Methicillin-resistant Staphylococcus aureus (MRSA) has emerged over the last decade as a common etiologic agent of nosocomial pneumonia, especially in intensive care units (ICUs). For example, the prevalence of MRSA as a cause of nosocomial pneumonia (NP) in intensive care units (ICU) has been reported as $37 \%$ in Germany, $54 \%$ in the US and $78 \%$ in Asia and Latin America [5]. Patients with diabetes appear to be at increased risk for acquiring S. aureus pneumonia [6-8], and patients requiring renal dialysis are at risk for hospital-acquired pneumonia, healthcare associated pneumonia and ventilator associated pneumonia caused by multi-drug resistant pathogens [8]. A recent study by Haque and colleagues found that 28-day mortality rates were higher among ICU patients with MRSA pneumonia when they had comorbid diabetes [9].

Current guidelines for the management of adults with hospital-acquired, ventilator-associated and healthcareassociated pneumonia issued jointly by the American Thoracic Society and the Infectious Diseases Society of America (IDSA) recommend either linezolid or vancomycin as appropriate antibiotic agents for the treatment of MRSA nosocomial pneumonia (NP) [8]. The guidelines do not, however, address the potential for worse outcomes of this infection in a patient with diabetes, nor how the presence of this comorbidity might affect selection of antibiotic therapy. Presumably, this is based on a lack of published data on these issues.

We hypothesized that the immune and lung dysfunction commonly associated with diabetes may lead to a worse response to antibiotic treatment for MRSA pneumonia among these patients compared with nondiabetic patients. Further, because there has been a consistent trend in published trials toward better outcomes with treatment of MRSA pneumonia with linezolid than with other antibiotic agents [10-12] we wished to investigate the potential effect of underlying diabetes on treatment outcomes with linezolid versus vancomycin. To evaluate our hypothesis we conducted a post-hoc, subgroup analysis of data taken from a recent large, multicenter, prospective, randomized controlled study to examine the effect of diabetes mellitus on clinical and microbiologic outcomes in patients treated with either linezolid or vancomycin for culture-confirmed MRSA NP [12].

\section{Methods}

We retrospectively analyzed data from a prospective, multicenter, international study comparing linezolid (600 mg twice a day) to vancomycin $(15 \mathrm{mg} / \mathrm{kg}$ twice a day, with dose adjustment as necessary based on trough levels and creatinine clearance) administered for 7-14 days for the treatment of NP confirmed by culture to be caused by MRSA. The previously published main study provides details of the study design and overall results [12]. The study was approved by an institutional review board or ethics committee at each investigational site. Investigators obtained written informed consent from each patient or legally authorized representative. Briefly, male and female patients $\geq 18$ years old were enrolled if they had NP (defined as at least two clinical signs and symptoms consistent with pneumonia [i.e., new or worsening cough or purulent sputum, auscultatory findings of pneumonia, dyspnea, tachypnea, hypoxemia or new respiratory failure requiring mechanical ventilation] acquired after $48 \mathrm{~h}$ in an inpatient healthcare or chroniccare facility, or after recent hospitalization [within 90 days] or undergoing chronic dialysis within 30 days), and a respiratory specimen for culture. Potential patients were not excluded based on their baseline blood glucose levels; no baseline hemoglobin A1c levels were recorded. Patients could receive up to two days of non-study antibiotic therapy prior to randomization or study entry.

A local, unblinded pharmacist prepared the study medication, monitored and adjusted vancomycin doses according to local protocols and measurements of trough vancomycin levels and renal function. The investigators and patients were blinded throughout the study. Pending culture results, all patients could initially receive an antibiotic with activity against aerobic gramnegative bacterial pathogens, but not one with activity against MRSA; mixed infections were treated with additional agents for gram-negative antibiotic coverage. Patients were clinically assessed at baseline, on day 3 and every 3 days during treatment. Respiratory cultures were repeated $48-72 \mathrm{~h}$ after treatment initiation, at end of treatment (EOT), and at end of study (EOS, defined as 7-30 days after EOT).

The current analysis included only the modifiedintent-to-treat (MITT) population, defined as patients who received at least one dose of study drug and had 
culture-proven MRSA pneumonia. Since we used previously collected data without personal identifiers for this secondary analysis we did not obtain a separate ethics consent. We classified patients into those with, and those without, diabetes mellitus, based on the data recorded by the investigator on the comorbidity case report forms provided for each enrolled patient. Clinical outcome was primarily assessed by the investigator within 5 days of EOT and at EOS, with occasional override by the sponsor based on the criteria of Additional file 1: Table S1. Any revisions in classification of outcome were made before unblinding. Microbiologic responses were determined at EOT and EOS, based on the results of repeat cultures obtained from the original infection site. Additional file 1: Table S1 provides the definitions of clinical and microbiologic outcomes. We also assessed all treatment-related adverse events (AE), serious adverse events (SAEs), AEs that led to study drug discontinuation, and day 28 and day 60 (all-cause) mortality.

\section{Statistical analysis}

To assess statistical differences in the distribution of baseline characteristics between the two treatment groups, we used one-way analysis of variance for continuous variables and Fisher's exact test or the chisquare test, as appropriate, for categorical variables. Prior to analysis of clinical and microbiologic response and mortality, a Breslow-Day test was conducted to evaluate the homogeneity of treatment for the nonrandomized diabetes subgroups. We excluded patients who were classified in the "unknown" (clinical) or "indeterminate" (microbiologic) categories from statistical analysis of response. To assess the association of variables with clinical response and mortality among patients with diabetes and MRSA NP, we performed multivariate logistic regression analyses. Prior to model building we took into consideration covariate reduction techniques: near zero variance, missingness and covariate correlations, associations and clusters. We identified variables for inclusion in the final multivariate logistic regression model based on selection in at least $50 \%$ of 1000 bootstrap samples, where the model for each bootstrap was selected by backward elimination with an alpha stay criterion of 0.05 . Baseline variables in the multivariate analysis included: type of antibiotic treatment; age; weight; sex; race; presence of infiltrate on chest $\mathrm{x}$-ray; presence of pleural effusion; Acute Physiology and Chronic Health Evaluation (APACHE) score at baseline; presence of bacteremia; ICU admission at baseline; pathogen type (cultures positive for MRSA alone or in conjunction with other gram-positive, gramnegative or anaerobic organism[s]); type of ward or service on which the patient was hospitalized (surgical, medical, vs trauma); admission from a long term care facility; presence of co-morbidities (e.g., neoplastic, renal/ urinary, pulmonary, hepatobiliary, vascular, gastrointestinal); medications at baseline; a history of undergoing renal dialysis; baseline blood glucose; smoking status (current, ex-smoker or nonsmoker); pneumonia type (ventilator-associated, hospital-associated or healthcare associated); and, minimum inhibitory concentration (MIC) of the organism against linezolid and vancomycin. We used 2-sided tests for all statistical comparisons and considered $p$-values $<0.05$ as statistically significant. For statistical procedures we used SAS, version 9.2 (SAS Institute, Cary, NC).

\section{Results}

\section{Study population and patient demographics}

Overall, 448 enrolled patients met the study entry criteria, 183 of whom had diabetes and 265 were nondiabetic. Among the patients with diabetes, 87 (47.5\%) were randomized to receive linezolid and 96 (52.5\%) to receive vancomycin. Among non-diabetic patients 137 $(51.7 \%)$ received linezolid and $128(48.3 \%)$ received vancomycin. The majority of patients with diabetes were insulin-treated at enrollment: $66.7 \%$ among the linezolid-treated and $77.1 \%$ among the vancomycintreated patients.

Demographic and baseline characteristics were similar between the linezolid and vancomycin treatment groups (Table 1). Compared to the non-diabetic patients, the diabetic patients had a significantly higher percent: older than 50 years of age; heavier than $75 \mathrm{~kg}$; with an APACHE II score $\geq 20$; or, with a cardiac, vascular, renal or gastrointestinal comorbidity (Table 1 ). The diabetic and non-diabetic groups were not statistically different in their baseline microbiological results, vancomycin MICs, clinical pulmonary infection scores, percent of patients who were ventilated or had bacteremia, frequency of pleural effusion (43.7 vs $45.7 \%, p=0.70$ ) or bilateral lung involvement on chest X-ray (72.1 vs. $66.4 \% p=$ 0.17 ).

The baseline non-fasting blood glucose levels were similar between the diabetic and non-diabetic linezolid treated patients (mean $\pm \mathrm{SD} 176.3 \pm 116.7 \mathrm{mg} / \mathrm{dL}$ vs. $164.7 \pm 152.7 \mathrm{mg} / \mathrm{dL}$ ), and non-diabetic vancomycin treated patients $(167.8 \pm 123.5 \mathrm{mg} / \mathrm{dL}$ for diabetic vs. $174.9 \pm 158.9 \mathrm{mg} / \mathrm{dL}$ ) (Table 1).

\section{Treatment outcomes}

The outcomes by diabetes subgroup are similar to those overall observed in the primary study, particularly in regards to EOS, EOT outcomes and mortality [12]. The duration of antibiotic therapy provided was similar for the two treatment arms and among the diabetic and non-diabetic patients (Table 1). On treatment days 3, 6 
Table 1 Demographic and clinical characteristics of diabetic and non-diabetic patients treated with either linezolid or vancomycin for MRSA-nosocomial pneumonia in the modified intent to treat (MITT) population

\begin{tabular}{|c|c|c|c|c|}
\hline \multirow[b]{2}{*}{ Characteristic } & \multicolumn{2}{|c|}{ Diabetic Patients $(N=183)$} & \multicolumn{2}{|c|}{ Non-diabetic Patients $(N=265)$} \\
\hline & Linezolid $N=87$ & Vancomycin $N=96$ & Linezolid N=137 & Vancomycin $N=128$ \\
\hline$\overline{\text { Age }(y), \text { mean }(S D) * * *}$ & $67.1(13.0)$ & $69.8(12.8)$ & $58.2(19.8)$ & $56.1(19.3)$ \\
\hline Male Sex, $n(\%)$ & $57(65.5)$ & $55(57.3)$ & $94(68.6)$ & $88(68.8)$ \\
\hline \multicolumn{5}{|l|}{ Race, $n(\%)$} \\
\hline White & $59(67.8)$ & $60(62.5)$ & $97(70.8)$ & $92(71.9)$ \\
\hline Black & $11(12.6)$ & $15(15.6)$ & $14(10.2)$ & $16(12.5)$ \\
\hline Asian & $12(13.8)$ & $17(17.7)$ & $20(14.6)$ & $16(12.5)$ \\
\hline Other & $5(5.7)$ & $4(4.2)$ & $6(4.4)$ & $4(3.1)$ \\
\hline Treatment duration (days), mean (SD) & $9.7(4.1)$ & $9.1(4.9)$ & $10.1(3.8)$ & $10.0(4.1)$ \\
\hline Baseline blood glucose (mg/dl), n⿳亠丷⿵冂⿰丨丨 & 83/176.3 (116.7) & $91 / 167.8(123.5)$ & 129/164.7 (152.7) & 121/174.9 (158.9) \\
\hline \multicolumn{5}{|l|}{ Bacteremia, $n(\%)$} \\
\hline Yes & $6(6.9)$ & $10(10.4)$ & $9(6.6)$ & $14(10.9)$ \\
\hline No & $78(89.7)$ & $85(88.5)$ & $116(84.7)$ & $110(85.9)$ \\
\hline Unknown & $3(3.4)$ & $1(1.0)$ & $12(8.8)$ & $4(3.1)$ \\
\hline Insulin at Baseline $n(\%)$ & $58(66.7)$ & $74(77.1)$ & N/A & N/A \\
\hline Ventilated at baseline, $n(\%)$ & $58(66.7)$ & $70(72.9)$ & $95(69.3)$ & $93(72.7)$ \\
\hline \multicolumn{5}{|l|}{ APACHE Score, $n(\%)^{* * *}$} \\
\hline$<20$ & $50(57.5)$ & $52(54.2)$ & $91(66.4)$ & $87(68.0)$ \\
\hline$\geq 20$ & $36(41.4)$ & $42(43.8)$ & $43(31.4)$ & $39(30.5)$ \\
\hline Unknown & $1(1.1)$ & $2(2.1)$ & $3(2.2)$ & $2(1.6)$ \\
\hline CPIS score, $\mathrm{n}^{\mathrm{a}} /$ mean (SD) Baseline & $56 / 9.6(2.2)$ & $63 / 9.5(2.1)$ & $89 / 9.5(2.1)$ & $91 / 9.2(2.4)$ \\
\hline \multicolumn{5}{|l|}{ Chest x-ray, $n(\%)^{b}$} \\
\hline Unilateral & $20(23.0)$ & $29(30.2)$ & $39(28.5)$ & $50(39.1)$ \\
\hline Bilateral & $65(74.7)$ & $67(69.8)$ & $98(71.5)$ & $78(60.9)$ \\
\hline Pleural effusion, $n(\%)$ & $42(48.3)$ & 38 (39.6) & $63(46.0)$ & $58(45.3)$ \\
\hline Weight (kg), $\mathrm{n}^{\mathrm{a}} /$ mean (SD) *** & $87 / 85.6(26.8)$ & 96/79.2 (22.4) & 137/73.2 (18.0) & $127 / 76.2(20.4)$ \\
\hline \multicolumn{5}{|l|}{ Comorbidities, n (\%) } \\
\hline Cardiac $* * *$ & $58(66.7)$ & 69 (71.9) & $73(53.3)$ & $68(53.1)$ \\
\hline Hepatobiliary & $13(14.9)$ & $10(10.4)$ & $21(15.3)$ & $21(16.4)$ \\
\hline Gastrointestinal *** & $57(65.5)$ & $56(58.3)$ & $71(51.8)$ & $63(49.2)$ \\
\hline Neoplastic & $8(9.2)$ & $13(13.5)$ & $12(8.8)$ & $12(9.4)$ \\
\hline Renal/Urinary *** & $40(46.0)$ & $53(55.2)$ & $43(31.4)$ & $41(32.0)$ \\
\hline Pulmonary & $65(74.7)$ & $67(69.8)$ & $87(63.5)$ & $87(68.0)$ \\
\hline Vascular *** & $38(43.7)$ & $37(38.5)$ & $38(27.7)$ & $41(32.0)$ \\
\hline \multicolumn{5}{|l|}{ Baseline pathogens, $n$ (\%) } \\
\hline MRSA only & $53(60.9)$ & $62(64.6)$ & $66(48.2)$ & $66(51.6)$ \\
\hline MRSA + other Gr $(+)$ & $6(6.9)$ & $9(9.4)$ & $16(11.7)$ & $9(7.0)$ \\
\hline MRSA + other Gr (-) & $19(21.8)$ & $18(18.8)$ & $41(29.9)$ & $38(29.7)$ \\
\hline MRSA+ other Gr (+) and Gr (-) & $5(5.7)$ & $3(3.1)$ & $8(5.8)$ & $10(7.8)$ \\
\hline MRSA + anaerobes & 0 & $1(1.0)$ & 0 & $1(0.8)$ \\
\hline MRSA + fungal & $4(4.6)$ & $3(3.1)$ & $6(4.4)$ & $4(3.1)$ \\
\hline \multicolumn{5}{|l|}{ Baseline Vancomycin MICs, n (\%) } \\
\hline$\leq 0.5$ & $7(8.0)$ & $2(2.1)$ & $10(7.3)$ & $12(9.4)$ \\
\hline 1 & $63(72.4)$ & $75(78.1)$ & $105(76.6)$ & $93(72.7)$ \\
\hline 2 & $7(8.0)$ & $7(7.3)$ & $6(4.4)$ & $11(8.6)$ \\
\hline 4 & 0 & $1(1.0)$ & 0 & $1(0.8)$ \\
\hline unknown & $10(11.5)$ & $11(11.5)$ & $16(11.7)$ & $11(8.6)$ \\
\hline
\end{tabular}

Abbreviations: CPIS clinical pulmonary infection score, MRSA methicillin-resistant S. aureus, SD standard deviation

${ }^{a}$ Denotes the number of patients with available data when different than the group total

${ }^{\mathrm{b}}$ Among diabetic patients treated with linezolid, 1 had a normal chest $x$-ray and one had unknown chest $x$-ray findings

${ }^{*} P<0.05$ for linezolid vs vancomycin treated diabetic patients; ${ }^{* *} P<0.05$ for linezolid vs vancomycin treated non-diabetic patients; ${ }^{* * *} P<0.05$ for diabetic patients vs. non-diabetic patients 
and 9 the vancomycin trough levels (in $03 \mathrm{BCg} / \mathrm{ml}$ median $[(\min , \max ])$ were $13.3(4.4,36.1), 15.0(6.4,41.4)$ and $18.0(4.9,26.2)$ among diabetic patients and 11.7 (2.8, 50.8), $14.7(2.7,45.0)$ and $13.7(2.0,46.9)$ among non-diabetic patients. The duration of ventilation and antibiotic treatment (data not shown), EOT and EOS overall clinical outcomes (Fig. 1) and microbiologic outcomes (Fig. 2) were similar between diabetic and nondiabetic groups.

Among the diabetic patients, clinical and microbiologic success rates at EOT and EOS were higher among linezolid treated patients as compared to those treated with vancomycin (Figs. 1 and 2). At EOT clinical success was noted in $82.4 \%$ of diabetic patients treated with linezolid versus $64.8 \%$ of diabetic patients treated with vancomycin (risk difference $[\mathrm{RD}], 95 \%$ confidence intervals [CI]: $17.6 \%$, [4.5, 30.7]). Similarly, at EOT microbiologic success was noted for $83.8 \%$ of diabetic patients treated with linezolid vs. $57.0 \%$ of diabetic patients treated with vancomycin (RD, 95 \% CI: 26.8 \% [13.7, 39.9]). At the EOS visit, clinical success was found in $57.6 \%$ of the diabetic patients treated with linezolid and $39.3 \%$ of those treated with vancomycin [RD, $95 \%$ CI: $18.2 \%(2.6,33.9)]$; EOS microbiologic success was noted for $58.9 \%$ of diabetic patients treated with linezolid and $41.1 \%$ of those treated with vancomycin (RD, $95 \%$ CI: $17.8 \%[2.6,33.0]$ ).
Among the non-diabetic patients, clinical and microbiologic success rates at EOT and EOS were similar between treatment groups, except for clinical response at EOT (Figs. 1 and 2). At EOT, clinical success was noted in $76.7 \%$ of non-diabetic patients treated with linezolid versus $59.2 \%$ of non-diabetic patients treated with vancomycin (risk difference [RD], $95 \%$ confidence intervals $[\mathrm{CI}]: 17.5 \%,[6.3,28.8])$.

A multivariate regression analysis was conducted to evaluate the association between baseline factors and clinical response at EOT and EOS among patients with diabetes (Table 2). The final multivariate model for clinical response at EOT had the following predictors: combined MIC; vasopressor use; smoking status; study drug, bacteremia; and, pneumonia type. The final multivariate model for clinical response at EOS had the following predictors: race; and, vasopressor use. Diabetic patients treated with linezolid were 2.8 times more likely to have clinical success at EOT than those treated with vancomycin $(p=0.02)$. Second, diabetic patients without bacteremia accompanying their NP were 5 times more likely to have clinical success at EOT compared to those with bacteremia $(p=0.009)$. At EOS diabetic patients who were not on vasopressors at baseline were 3.3 times as likely to have clinical success compared to those who were, and Asian diabetic patients were almost 5 times

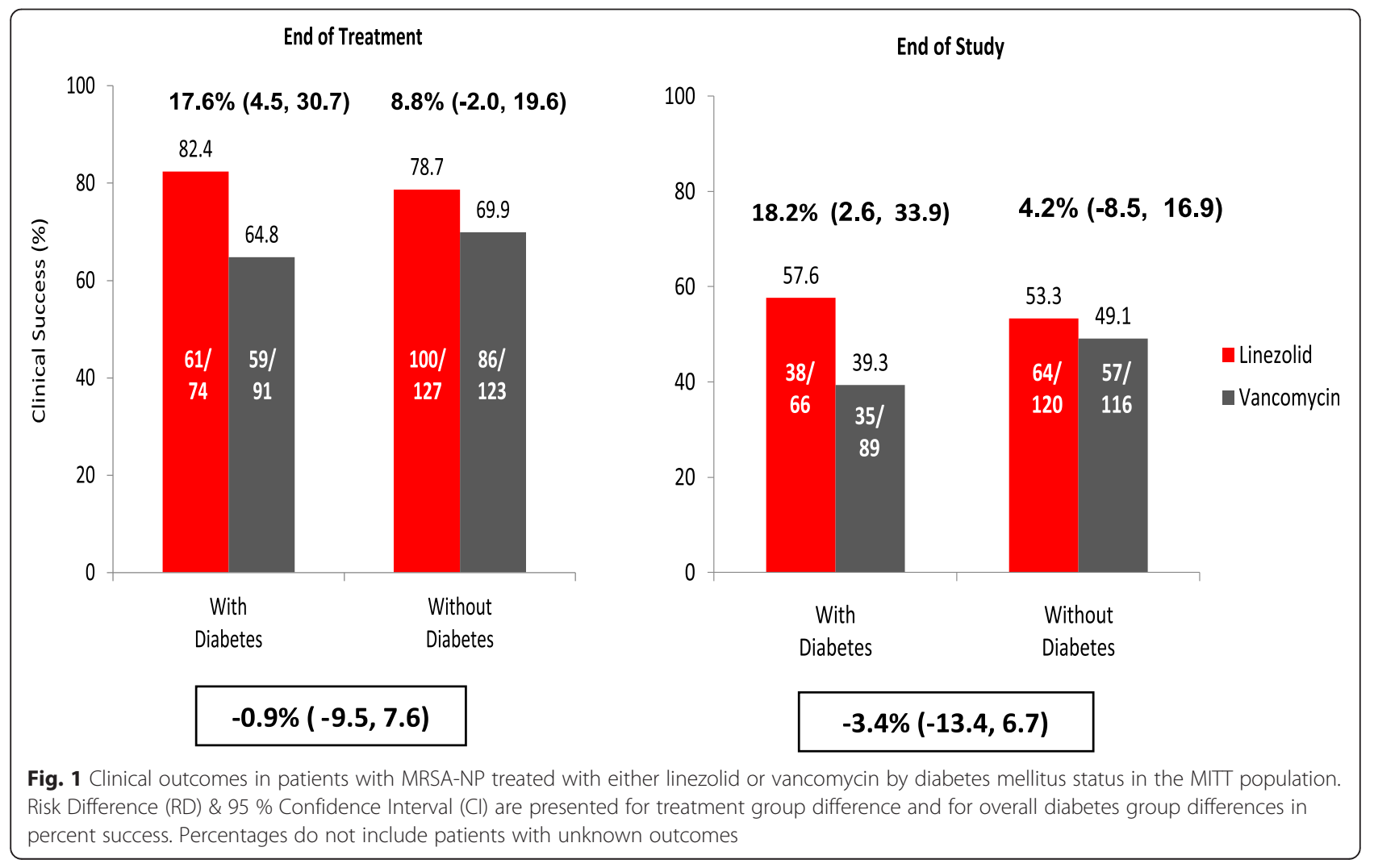




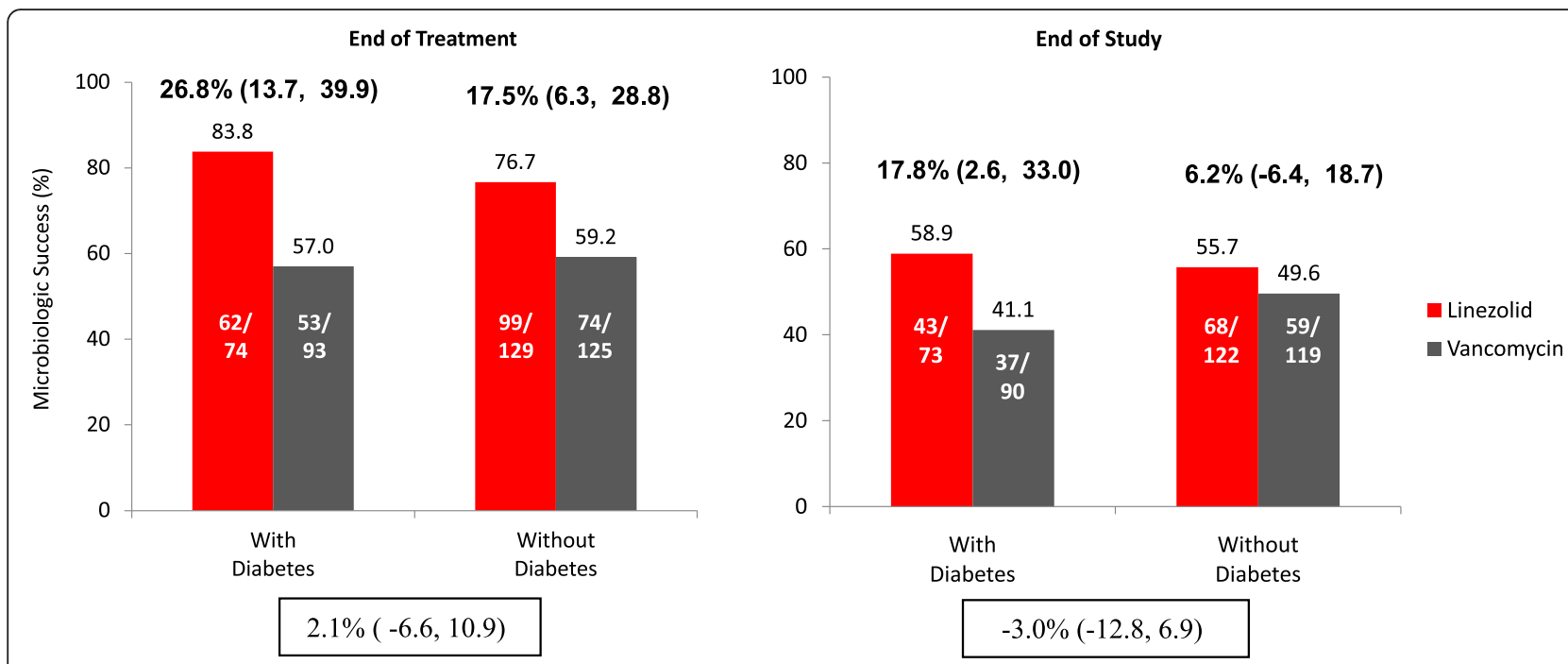

Fig. 2 Microbiologic outcomes in patients with MRSA-NP treated with either linezolid or vancomycin by diabetes mellitus status in the MITT population. Risk Difference (RD) \& $95 \%$ Confidence Interval (Cl) are presented for treatment group difference in percent success. Percentages do not include patients with unknown outcomes

more likely to have clinical success at EOS compared with white diabetic patients.

All-cause mortality within 28 days after randomization was higher among diabetic patients compared to nondiabetic patients ( 23.5 vs $14.7 \%, \mathrm{RD}=8.8 \%, 95 \% \mathrm{CI}$ $[1.4,16.3])$, but was similar for linezolid and vancomycin treated diabetic patients $(20.7 \%$ for linezolid versus $26.0 \%$ for vancomycin) and non-diabetic patients (17.5\% for linezolid versus $11.7 \%$ for vancomycin). Results for all-cause mortality within 60 days after randomization were non-significantly higher among diabetic patients compared to non-diabetic patients [29.5 vs $23.0 \%, \mathrm{RD}=6.5 \%, 95 \% \mathrm{CI}(-1.8,14.8)]$. Adverse events resulting in death that were reported for more diabetic patients compared to non-diabetic patients were cardiovascular and cerebrovascular events, respiratory arrest and septic shock. Among diabetic patients, pneumonia, as an adverse event resulting in death, was reported for more linezolid-treated patients compared to vancomycin-treated patients. Adverse events resulting in death reported for more vancomycin-treated diabetic patients than linezolid-treated diabetic patients were multi-organ failure, respiratory failure, sepsis and septic shock.

A multivariate regression analysis was conducted to evaluate the association between baseline factors and day 28 mortality among patients with diabetes (Table 2). The final multivariate model for mortality had the following predictors: age; history of chronic renal dialysis; race; hospitalization service type; bacteremia; and, pleural effusion. There was a six-fold increased risk of death within 28 days of randomization among diabetic patients who were receiving chronic renal dialysis and a significantly increased risk of death with increasing age (a 1.4-fold increase for every 10 year increase in age). Furthermore, diabetic patients with pleural effusion were half as likely to die compared to diabetic patients without pleural effusion. Asian diabetic patients were 10 times less likely to die compared to white diabetic patients.

\section{Safety}

Rates of treatment-related adverse events, as well as study drug discontinuations due to adverse events, were similar for diabetic and non-diabetic patients in the MITT population. The percentages of treatment-related adverse events, and study drug discontinuations were also similar between the two drug treatment groups (Table 3).

\section{Discussion}

Patients with diabetes mellitus have been reported to be at high risk for colonization and several types of infection with MRSA, especially pneumonia and soft tissue infections [13-17]. Furthermore, some previous studies suggest that diabetic patients with complicated MRSA skin and soft tissue infections respond less well to treatment compared to non-diabetic patients [18-20]. In a diabetic mouse model, treatment with either systemic linezolid or daptomycin had a more rapid therapeutic effect compared with vancomycin, but after day 1, all three antibiotics had similar efficacy against MRSA wound infection [21]. A study looking at the effect of the addition of a macrolide antibiotic to initial therapy with a third generation-beta lactam among patients 
Table 2 Multivariate logistic regression modelling in patients with diabetes

\begin{tabular}{|c|c|c|c|}
\hline Variable & Adjusted OR & $95 \% \mathrm{Cl}$ & $P$ value \\
\hline \multicolumn{4}{|l|}{ Clinical Success (mITT Population) } \\
\hline \multicolumn{4}{|l|}{ End of Treatment } \\
\hline Vasopressors at Baseline (Yes vs No) & 0.4 & $(0.2,1.1)$ & 0.073 \\
\hline Treatment (LZD vs. VAN) & 2.8 & $(1.2,6.8)$ & 0.022 \\
\hline Bacteremia (Yes vs No) & 0.2 & $(0.1,0.7)$ & 0.009 \\
\hline \multicolumn{4}{|l|}{ Combined MIC (vs. 0.5) } \\
\hline 1 & 1.5 & $(0.2,9.1)$ & 0.673 \\
\hline 2 or 4 & 0.4 & $(0.1,3.2)$ & 0.384 \\
\hline \multicolumn{4}{|l|}{ Pneumonia Type } \\
\hline HCAP vs. HAP & 2.9 & $(0.6,13.7)$ & 0.176 \\
\hline VAP vs. HAP & 0.6 & $(0.2,1.5)$ & 0.239 \\
\hline HCAP vs. VAP & 5.2 & $(1.3,21.6)$ & 0.023 \\
\hline \multicolumn{4}{|l|}{ Smoking Status } \\
\hline Current vs. Non-Smoker & 0.9 & $(0.3,2.4)$ & 0.774 \\
\hline Ex vs. Non-Smoker & 2.3 & $(0.8,6.1)$ & 0.105 \\
\hline \multicolumn{4}{|l|}{ End of Study } \\
\hline Vasopressors at Baseline & 0.3 & $(0.1,0.8)$ & 0.019 \\
\hline \multicolumn{4}{|l|}{ Race (vs White) } \\
\hline Asian & 4.9 & $(1.7,13.6)$ & 0.002 \\
\hline Black & 1.7 & $(0.7,4.3)$ & 0.281 \\
\hline Other & 1.1 & $(0.2,5.5)$ & 0.885 \\
\hline \multicolumn{4}{|l|}{ Day 28 Mortality (mlTT population) } \\
\hline Age (10 years) & 1.4 & $(1.1,1.9)$ & 0.014 \\
\hline Chronic Dialysis Care (Yes vs No) & 5.9 & $(1.8,19.6)$ & 0.004 \\
\hline \multicolumn{4}{|l|}{ Race (vs. White) } \\
\hline Asian & 0.1 & $(0.0,0.5)$ & 0.004 \\
\hline Black & 1.0 & $(0.4,2.6)$ & 0.987 \\
\hline Other & 0.8 & $(0.1,3.9)$ & 0.735 \\
\hline \multicolumn{4}{|l|}{ Subject Type (vs. Medical) } \\
\hline Surgical & 1.0 & $(0.4,2.2)$ & 0.921 \\
\hline Trauma & 1.3 & $(0.4,5.1)$ & 0.665 \\
\hline Bacteremia (Yes vs. No) & 2.2 & $(0.8,6.0)$ & 0.115 \\
\hline Pleural Effusion (Yes vs. No) & 0.5 & $(0.2,1.0)$ & 0.040 \\
\hline
\end{tabular}

$\mathrm{Cl}$ confidence interval, $\mathrm{m} / \mathrm{T}$ modified intent-to-treat $O R$ odds ratio

with community acquired pneumonia did not show a difference between diabetic and non-diabetic subgroups [22].

We are not aware of any studies similar to ours on the role of co-morbid diabetes mellitus in patients with nosocomial pneumonia. A systematic review and meta-analysis published in 2013 found 9 studies comparing treatment of hospital-acquired pneumonia (of any cause) with linezolid versus vancomycin [23]. The results suggested that the two drugs had similar efficacy and safety profiles, including for infections caused by MRSA, but there was no breakdown in results based on the presence of diabetes.
Thus, we conducted an analysis of data obtained from a recent large, randomized, prospective, multi-national clinical trial of culture confirmed MRSA NP. Our results show that treatment of diabetic patients with linezolid resulted in higher clinical and microbiologic success rates at EOT and EOS compared to treatment with vancomycin. In contrast, there were no significant differences for clinical and microbiologic success rates between the treatment arms among non-diabetic patients.

It would be reasonable to expect the random blood glucose levels to be higher in the diabetic patients, but 
Table 3 Adverse events among diabetic and non-diabetic patients in the modified-intent-to-treat population

\begin{tabular}{|c|c|c|c|c|}
\hline \multirow[b]{2}{*}{ Adverse event (AE) n (\%) } & \multicolumn{2}{|c|}{ Diabetic Patients $(N=183)$} & \multicolumn{2}{|c|}{ Non-diabetic Patients $(N=265)$} \\
\hline & Linezolid $N=87$ & Vancomycin $N=96$ & Linezolid $N=137$ & Vancomycin $N=128$ \\
\hline Patients with a treatment- related $\mathrm{AE}$ & $22(25.3)$ & $26(27.1)$ & $46(33.6)$ & $43(33.6)$ \\
\hline Patients with a serious $A E$ & $33(37.9)$ & $45(46.9)$ & $56(40.9)$ & $36(28.1)$ \\
\hline Patients with study drug discontinuation due to $\mathrm{AE}$ & $3(3.4)$ & $8(8.3)$ & $9(6.6)$ & $9(7.0)$ \\
\hline \multicolumn{5}{|l|}{ Patients with specific AEs: } \\
\hline Diarrhea & $12(13.8)$ & $14(14.6)$ & $28(20.4)$ & $20(15.6)$ \\
\hline $\operatorname{Rash}^{\mathrm{a}}$ & $6(6.9)$ & $8(8.3)$ & $16(11.7)$ & $9(7.0)$ \\
\hline Nausea & $7(8.0)$ & $7(7.3)$ & $10(7.3)$ & $11(8.6)$ \\
\hline Vomiting & $3(3.4)$ & $4(4.2)$ & $8(5.8)$ & $8(6.3)$ \\
\hline Thrombocytopenia $^{\mathrm{d}}$ & $17(20.7)$ & $13(14.3)$ & $18(13.5)$ & $15(12.3)$ \\
\hline Kidney impairment ${ }^{\mathrm{b}}$ & $3(3.4)$ & $13(13.5)$ & $5(3.6)$ & $9(7.0)$ \\
\hline IV site complications ${ }^{c}$ & $3(3.4)$ & $5(5.2)$ & $6(4.4)$ & $6(4.7)$ \\
\hline
\end{tabular}

a Rash includes the following AE preferred terms: Genital rash, Rash, Rash erythematous, Rash generalized, Rash macular, Rash maculo-papular, Rash popular and Rash pustular

${ }^{\mathrm{b}}$ Kidney Impairment includes the following AE preferred terms: Renal failure, Renal failure acute, Renal failure chronic and Renal impairment

c IV Site Complications include the following AE preferred terms: Catheter site erythema, Catheter site haematoma, Catheter site haemorrhage, Catheter site infection, Catheter site pain, Infusion site cellulitis, Infusion site erythema, Infusion site extravasation, Infusion site infection, Infusion site phlebitis and Infusion site thrombosis

${ }^{\mathrm{d}}$ Thrombocytopenia was defined as a platelet count of $<150,000 \mu \mathrm{L}$, if platelet count was normal at baseline, or a $50 \%$ drop if platelet count was not normal at baseline

they were not in this study. This may be because the diabetic patients had received prior treatment for glycemic control. In addition, the elevated blood glucose levels in the non-diabetic patients could at least be partly attributable to the stress of acute illness. It is also possible that some "non-diabetics" may be undiagnosed diabetics (or have pre-diabetes).

The concentration of vancomycin in lung epithelial lining fluid (ELF) is dependent upon blood vancomycin levels and alveolar capillary membrane protein permeability [18, 24], and is an important determinant of vancomycin antibacterial activity against MRSA in the lung [25]. Linezolid has been shown to have better ELF penetration than vancomycin in animal models of MRSA NP [26]. A meta-analysis of pulmonary function found that patients with diabetes have higher rates of restrictive lung disease [4], and postmortem histologic examination of lung tissue from patients with diabetes mellitus showed thickening of alveolar epithelial and pulmonary capillary basal laminae [27]. In our review of the literature we found no data on vancomycin ELF concentrations in patients with diabetes mellitus. In this double-blind clinical trial vancomycin trough levels were monitored and adjusted by local pharmacists and were similar between patients with and without diabetes mellitus; however, due to underlying lung pathology it may be that vancomycin penetration into pulmonary sites of infection is reduced, lessening the likelihood of cure of infection.

In our study the 28 day mortality rate for patients who had MRSA NP was higher among diabetic than nondiabetic patients, regardless of the treatment arm. A study by Falguera and colleagues reported an increased mortality among diabetic patients with communityacquired pneumonia that they attributed to underlying conditions, such as neoplastic disease, congestive heart failure, cerebrovascular disease, chronic renal disease, chronic obstructive pulmonary disease, chronic liver disease and HIV infection [28]. Patients with diabetes have also been shown to be at higher risk than non-diabetics for MRSA bacteremia [13, 29, 30]. In our study, the frequencies of multi-lobar pneumonia, pleural effusion and MRSA bacteremia were similar in the diabetic and nondiabetic patients with MRSA-NP. Perhaps not surprisingly, increasing age and undergoing chronic renal dialysis were associated with an increased mortality risk among diabetic patients. We also observed that the occurrence of cardiovascular and cerebrovascular events, as well as respiratory failure and septic shock, were events resulting in death more frequently reported for diabetic patients compared to non-diabetic patients. Of note, diabetic patients who were Asian, or who had pleural effusion at baseline were less likely to die from their MRSA-NP. Although recent studies suggest that body fat and lean mass content and fat distribution are different in Asians, and these differences may potentially lead to heterogeneity in the clinical presentation of agerelated chronic diseases between ethnicities [31], we cannot assess the effect of race on treatment outcome from this study.

Our study has several limitations. First, it was based on a retrospective review of prospectively obtained data and was not planned a priori. Second, it is a subgroup analysis with a relatively small sample size in some 
groups, therefore making it difficult to identify small but potentially clinically important differences. Third, we conducted an interaction test that showed no significant differences in treatment responses between the diabetic and non-diabetic patients. Fourth, the diagnosis of diabetes in enrolled patients was based on information provided by the investigators. We had no data on the patient's baseline hemoglobin A1C levels, which may have helped elucidate the relationship of prior glycemic control among patients with diabetes in the outcome of these infections. Additionally, diabetes mellitus is a disorder with a broad spectrum of severity. Identifying the type of diabetes ( 1 versus 2 ) and measurements of glycemic control (serum glucose or glycosylated hemoglobin) have limited ability to define the severity of the disease. As such, results should be interpreted with caution and considered as hypothesis-generating, rather than confirmatory. Given that treatment responses may differ between diabetic and non-diabetic patients, investigators in future clinical trials may wish to consider whether diabetic, pre-diabetic and non-diabetic groups should be stratified prior to randomization.

\section{Conclusions}

A key finding in our study was that among diabetic patients with MRSA NP the cure rate was significantly higher among those treated with linezolid compared with those treated with vancomycin. Regardless of treatment arm, the 28-day mortality rate was higher among patients with diabetes than among non-diabetics, but were similar for linezolid and vancomycin treated diabetic patients. Increased mortality among the patients with diabetes mellitus was associated with increasing age and having received chronic renal dialysis. If our findings are replicated by other investigators, clinicians might consider treating diabetic patients with MRSA-NP with linezolid.

\section{Additional file}

Additional file 1: Definitions of Clinical and Microbiologic Outcomes. Additional file 1: Table S1. defines the clinical outcomes [clinical cure, clinical improvement (only at EOT), clinical failure and indeterminate] and microbiologic outcomes [documented microbiologic eradication, presumed microbiologic eradication, presumed microbiologic persistence, superinfection, colonization and indeterminate]. (DOCX $14 \mathrm{~kb}$ )

\section{Acknowledgments}

The authors thank Vito Calamia and Dianne Ruzzi for their programming support.

\section{Funding}

This study was sponsored by Pfizer Inc. The study was conceived by OE who is a Pfizer employee. MW is also a Pfizer employee, CD was a Pfizer employee at the time this study was conceived, he currently is not.
All authors participated in the design of the study, data collection, analyses and interpretation of data and in writing the manuscript.

\section{Availability of data and materials}

All the data supporting our findings is contained within the manuscript, and no identifying/confidential patient data will be shared.

\section{Authors' contributions}

All authors participated in the design of the study and manuscript development. MW performed the statistical analysis. OE conceived of the study, and led the design, coordination and manuscript development. All authors read and approved the final manuscript.

\section{Competing interests}

Dr. Lipsky has served as a consultant and advisory board member for Pfizer; he received no remuneration for the development of this manuscript. Dr. da Costa was an employee of Pfizer. He was not paid for the development of the manuscript. Ozlem Equils, MD and Michele Wible are employees of Pfizer Inc., they are salaried by Pfizer and own small amount of Pfizer stock. Statistical support was provided by Amy Stein of Quintiles Inc. and was funded by Pfizer Inc.

\section{Consent for publication}

Not applicable.

\section{Ethics approval and consent to participate}

An institutional review board or ethics committee at each investigational site approved the study. Investigators obtained written informed consent from each patient or legally authorized representative. Previously collected data was utilized for this secondary analysis and a separate ethics consent was not obtained.

\section{Author details}

${ }^{1}$ Pfizer Inc., Collegeville, PA, USA. ${ }^{2}$ University of Oxford, Oxford, UK. ${ }^{3}$ University of Washington, Seattle, WA, USA.

Received: 29 June 2015 Accepted: 15 August 2016

Published online: 06 September 2016

\section{References}

1. Geerlings SE, Hoepelman AIM. Immune dysfunction in patients with diabetes. FEMS Immunol Med Microbiol. 2000;26:259-66.

2. Koziel H, Koziel MJ. Pulmonary complications of diabetes mellitus. Infect Dis Clin North Am. 1995;9:65-96.

3. Kabitz HJ, Sonntag F, Walker D, et al. Diabetic polyneuropathy is associated with respiratory muscle impairment in type 2 diabetes. Diabetologia. 2008:511:191-7.

4. van den Borst B, Gosker HR, Zeegers MP, Schols AM. Pulmonary function in diabetes: a metaanalysis. Chest. 2010;138:393-406.

5. Meyer E, Schwab F, Gastmeier P. Nosocomial methicillin resistant Staphylococcus aureus pneumonia - epidemiology and trends based on data of a network of 586 German ICUs (2005-2009). Eur J Med Res. 2010;15:514-24.

6. Joshi N, Caputo GM, Weitekamp MR, Karchmer AW. Infections in patients with diabetes mellitus. N Engl J Med. 1999:341:1906-12.

7. Muller LM, Gorter KJ, Hak E, Goudzwaard WL, Schellevis FG, Hoepelman Al, Rutten GE. Increased risk of common infections in patients with type 1 and type 2 diabetes mellitus. Clin Infect Dis. 2005:41:281-8.

8. Niederman MS, Craven DE, Bonten MJ, American Thoracic Society, The Infectious Diseases Society of America, et al. Guidelines for the management of adults with hospital-acquired, ventilator-associated, and healthcare-associated pneumonia. Am J Respir Crit Care Med. 2005;171:388-416.

9. Haque NZ, Arshad S, Peyrani P, Ford KD, Perri MB, Jacobsen G, Reyes K, Scerpella EG, Ramirez JA, Zervos MJ. Analysis of Pathogen and host factors related to clinical outcomes in patients with hospital-acquired pneumonia due to methicillin-resistant staphylococcus aureus. J Clin Microbiol. 2012;50:1640-4

10. Wunderink RG. How important is methicillin-resistant Staphylococcus aureus as a cause of community-acquired pneumonia and what is best antimicrobial therapy? Infect Dis Clin North Am. 2013;27:177-88. 
11. Peyrani P, Wiemken TL, Kelley R, Zervos MJ, Kett DH, File Jr TM, Stein GE, Ford KD, Scerpella EG, Welch V, Ramirez JA. IMPACT-HAP Study Group Higher clinical success in patients with ventilator-associated pneumonia due to methicillin-resistant Staphylococcus aureus treated with linezolid compared with vancomycin: results from the IMPACT-HAP study. Crit Care. 2014;18:R118.

12. Wunderink RG, Niederman MS, Kollef MH, Shorr AF, Kunkel MJ, Baruch A McGee WT, Reisman A, Chastre J. Linezolid in methicillin-resistant Staphylococcus aureus nosocomial pneumonia: a randomized, controlled study. Clin Infect Dis. 2012;54:621-8.

13. Breen JD, Karchmer AW. Staphylococcus aureus infections in diabetic patients. Infect Dis Clin North Am. 1995;9:11-24.

14. McKinnell JA, Miller LG, Eells SJ, Cui E, Huang SS. A systematic literature review and meta-analysis of factors associated with methicillin-resistant Staphylococcus aureus colonization at time of hospital or intensive care unit admission. Infect Control Hosp Epidemiol. 2013;34:1077-86.

15. Shorr AF, Myers DE, Huang DB, Nathanson BH, Emons MF, Kollef MH. A risk score for identifying methicillin-resistant Staphylococcus aureus in patients presenting to the hospital with pneumonia. BMC Infect Dis. 2013;13:268-79.

16. Chen CC, Pass SE. Risk factors for and impact of methicillin-resistant Staphylococcus aureus nasal colonization in patients in a medical intensive care unit. Am J Infect Control. 2013:41:1100-11.

17. Korol E, Johnston K, Waser N, Sifakis F, Jafri HS, Lo M, Kyaw MH. A systematic review of risk factors associated with surgical site infections among surgical patients. PLoS One. 2013;8:e83743.

18. Lipsky BA, Itani KM, Weigelt JA, Joseph W, Paap CM, Reisman A, Myers DE, Huang DB. The role of diabetes mellitus in the treatment of skin and skin structure infections caused by methicillin-resistant Staphylococcus aureus: results from three randomized controlled trials. Int J Infect Dis. 2011;15:e140-6.

19. Haque NZ, Arshad S, Peyrani P, Ford KD, Perri MB, Jacobsen G, Reyes K, Scerpella EG, Ramirez JA, Zervos MJ. Analysis of pathogen and host factors related to clinical outcomes in patients with hospital-acquired pneumonia due to methicillin-resistant Staphylococcus aureus. J Clin Microbiol. 2012;50:1640-4

20. Polverino E, Torres A. Current perspective of the HCAP problem: is it CAP or is it HAP? Semin Respir Crit Care Med. 2009;30:239-48.

21. Guo Y, Ramos Rl, Cho JS, Donegan NP, Cheung AL, Miller LS. In vivo bioluminescence imaging to evaluate systemic and topical antibiotics against community-acquired methicillin-resistant Staphylococcus aureus-infected skin wounds in mice. Antimicrob Agents Chemother. 2013;57:855-63.

22. Burgess DS, Lewis 2 nd JS. Effect of macrolides as part of initial empiric therapy on medical outcomes for hospitalized patients with communityacquired pneumonia. Clin Ther. 2000;22:872-8.

23. Kalil AC, Klompas M, Haynatzki G, Rupp ME. Treatment of hospital-acquired pneumonia with linezolid or vancomycin: a systematic review and metaanalysis. BMJ Open. 2013;3:e003912.

24. Lamer C, de Beco V, Soler P, Calvat S, Fagon JY, Dombret MC, Farinotti R, Chastre J, Gibert C. Analysis of Vancomycin entry into pulmonary lining fluid by bronchoalveolar lavage in critically ill patients. Antimicrob Agents Chemother. 1993:37:281-6.

25. Harigaya Y, Bulitta JB, Forrest A, Sakoulas G, Lesse AJ, Mylotte JM, Tsuji BT. Pharmacodynamics of Vancomycin at simulated epithelial lining fluid concentrations against methicillin-resistant Staphylococcus aureus (MRSA): implications for dosing in MRSA pneumonia. Antimicrob Agents Chemother. 2009;53:3894-901.

26. Martinez-Olondris P, Rigol M, Soy D, Guerrero L, Agusti C, Quera MA, Li Bassi G, Esperatti M, Luque N, Liapikou M, Filella X, Marco F, de la Bellacasa JP, Torres A. Efficacy of linezolid compared to vancomycin in an experimental model of pneumonia induced by methicillin-resistant Staphylococcus aureus in ventilated pigs. Crit Care Med. 2012:40:162-8.

27. Vracko R, Thorning D, Huang TW. Basal lamina of alveolar epithelium and capillaries: quantitative changes with aging and in diabetes mellitus. Am Rev Respir Dis. 1979;120:973-83.

28. Falguera M, Pifarre R, Martin A, Sheikh A, Moreno A. Etiology and outcome of community-acquired pneumonia in patients with diabetes mellitus. Chest. 2005;128:3233-9.

29. Kaasch AJ, Barlow G, Edgeworth JD, Fowler Jr VG, Hellmich M, Hopkins S, Kern W, Llewelyn MJ, Rieg S, Rodriguez-Baño J, Scarborough M, Seifert H, Soriano A, Tilley R, Tőrők ME, Weiß V, Wilson AP, Thwaites GE, ISAC, INSTINCT, SABG, UKCIRG, Colleagues. Staphylococcus aureus bloodstream infection: a pooled analysis of five prospective, observational studies. J Infect. 2014;68:242-51.
30. Wiese L, Mejer N, Schønheyder HC, Westh H, Jensen AG, Larsen AR, Skov R, Benfield T, Danish Staphylococcal Bacteraemia Study Group. A nationwide study of comorbidity and risk of reinfection after Staphylococcus aureus bacteraemia. J Infect. 2013:67:199-205.

31. Haldar S, Chia SC, Henry CJ. Body composition in asians and caucasians: comparative analyses and influences on cardiometabolic outcomes. Adv Food Nutr Res. 2015;75:97-154.

\section{Submit your next manuscript to BioMed Central and we will help you at every step:}

- We accept pre-submission inquiries

- Our selector tool helps you to find the most relevant journal

- We provide round the clock customer support

- Convenient online submission

- Thorough peer review

- Inclusion in PubMed and all major indexing services

- Maximum visibility for your research

Submit your manuscript at www.biomedcentral.com/submit
) Biomed Central 